\title{
The dynamics of the development of mathematics skills: A comparison of theories of developing intelligence.
}

\author{
Abe D. Hofman ${ }^{* 1}$, Rogier A. Kievit ${ }^{2}$, Claire E. Stevenson ${ }^{1}$, Dylan Molenaar ${ }^{1}$, Ingmar \\ Visser $^{1}$, and Han L. J. van der Maas ${ }^{1}$ \\ ${ }^{1}$ University of Amsterdam \\ ${ }^{2} \mathrm{MRC}$ Cognition and Brain Sciences Unit, University of Cambridge
}

February 28, 2018

\begin{abstract}
This study investigates why children who are good at one math skill also perform well on other math tasks and seeks a solution by comparing two influential theories of general intelligence. The $g$-factor theory ( $g$ for 'general intelligence') implies a general math ability that steers all math-related development. In contrast, mutualism theory states that different math skills co-develop, positively influencing each others growth. We examined this with a large longitudinal data set $(N \approx 12.000)$ that tracked the development of basic (counting $\leftrightarrow$ addition) and more advanced (multiplication $\leftrightarrow$ division) math skills for a full school year. We used bivariate latent change score models to investigate whether $g$-factor or mutualism theory provides a better explanation of their co-development. We found that basic and more advanced math skills become more strongly related over time and that co-development is mutually beneficial. Both results support mutualism theory, a dynamic network perspective of cognitive development, where, in this case, growth in a particular math domain positively influences that of other math skills. Our results perhaps reveal the tip of the iceberg when it comes to the intricacies of co-developing math abilities. We discuss the implications of mutualism theory for understanding the dynamics of learning mathematics.
\end{abstract}

\section{Keywords}

Cognitive development; Longitudinal modeling; Mathematical abilities; Mutualism; Dynamic modeling; Networks

\section{Introduction}

The ability to do math is essential to daily life and the study of how this develops is of great interest in the fields of education and cognitive development (Siegler and Lortie-Forgues, 2014). Individual differences in mathematical ability relate to a wide set of cognitive abilities (Murnane et al., 1995) and lifespan outcomes (Siegler et al. 2012). Understanding how different mathematical abilities co-develop is crucial for understanding learning in general, and mathematics in particular. A large body of studies have focused on the symbiotic development of different cognitive and math skills, focusing particularly on positive correlations between domains (e.g., Geary et al., 2004: Halberda et al., 2008).

However, these cross-sectional studies can only provide indications of processes that occur within individuals over time (Tucker-Drob, 2009), and could possibly be misleading (Wohlwill, 1973; Molenaar, 2004). More

\footnotetext{
*The corresponding author is Abe Hofman, University of Amsterdam, Department of Psychological Methods, Nieuwe Achtergracht 129-B, 1018 WT Amsterdam. Funding by NWO (The Netherlands Organisation for Scientific Research) grant number 406-11-163.
} 
direct tests of how the development of different skills unfolds can be better established with a longitudinal approach (see for example the work of Geary et al. (1991) and van der Ven et al. (2012)). Currently, the dynamic processes that drive the development of mathematical abilities are unclear, as are possible direct links with the development of other skills. In this paper, we borrow ideas from intelligence research to investigate this issue.

The empirical finding of a positive correlational structure of individual differences in abilities across domains abilities is not unique to the field of mathematics. In the field of intelligence this is one of the most famous findings in cognitive psychology, and called the positive manifold(Spearman, 1927). This implies that people who perform well on one cognitive task also tend to score well on other cognitive tasks. In intelligence data, the correlational structure is often explained by $g$, for 'general intelligence', such that the positive correlational structure between the different cognitive test scores is modelled by a latent factor that represents general abilities across domains. Although $g$ is a useful construct in predicting educational success and other life outcomes, the presence of such a statistical factor does not necessarily imply a causal role for a single underlying factor across different cognitive tests (van der Maas et al., 2006, 2014b, 2017, Kruis and Maris, 2016). Nor does it imply that $g$ necessarily has an ontological status beyond a statistical entity (Borsboom et al., 2003). This follows from the fact that different hypotheses about the nature of the positive manifold can result in the same cross-sectional correlational data (Bartholomew et al., 2009, Anderson, 2017). In order to differentiate between different explanations of the positive manifold, hence to shed light on its true nature, a longitudinal approach is valuable (van der Maas et al., 2006, Molenaar, 2004).

In the current paper, following the approach of (Kievit et al., 2017b), we compare two important theories of the positive manifold based on their main distinguishing feature - the dynamics between different abilities over time. Using this approach, we investigate whether a mathematical $g$-factor (a general math ability) plays a causal role during development or whether bidirectional mutually beneficial relations between domains are sufficient. To this end we use a large longitudinal data set collected with an online learning program for mathematical ability in schoolchildren (Math Garden; Klinkenberg et al., 2011; Straatemeier, 2014). In this learning program children can log in at any time and play one or more games as often as they like. Increasing accessibility to Internet and the growing popularity of online learning systems provides large amounts of data on learning. Furthermore, the data we present was collected in a school setting and therefore captures learning in this naturalistic setting. This data set provides a unique sample to investigate the developmental patterns involved in learning mathematics.

Few studies have approached the study of the nature of the correlational structure between different skills from the perspective of the positive manifold and compared competing models of development. Even studies that have focused on longitudinal co-development of cognitive abilities have focused on development between domains such as memory, reasoning, vocabulary and perceptual speed (e.g., McArdle et al., 2002, Kievit et al. 2017b). No studies to date have examined whether a $g$-factor or interactive account better explains fine-grained development within a domain.

\subsection{Two different theories of cognitive development}

The robust finding of the positive manifold in scores on intelligence and mathematical abilities requires an explanation. The most prominent explanation is the $g$-factor theory itself (Jensen, 1998). This theory suggests that the latent factor (for general intelligence or in our case general mathematical ability) is of a natural kind and directly affects the scores on different domains that reflect, to varying extents, the general domain. This $g$ is the real variable of interest, an entity that exists independent of the data.

Although the standard $g$-factor theory does not include a developmental perspective van der Maas et al., 2006), a natural developmental interpretation of $g$ suggests that changes in $g$ induce changes in different subdomains. Thus changes in different skills, that function as indicators of $g$, should be caused by developmental processes in $g \rrbracket^{1}$ Therefore, it is often assumed that the correlational structure - reflecting the strength of the general factor - is relatively stable over time (Jensen, 1998, Gignac, 2014). Empirical results

\footnotetext{
${ }^{1}$ A strict interpretation of the $g$-factor model would even predict that development in a lower order factor would not result in any changes in other lower order factors, and would merely result in a larger residual variance (van der Maas et al., 2014a).
} 
on these differentiation effects are mixed (Molenaar et al., 2010). Gignac (2014) found that the strength of $g$ was relatively stable between ages 2.5 and 90. Whereas McArdle et al. (2002) concluded that the development of a single $g$-factor provides an overly simplistic view, which was based on the analyses of learning curves of 5 to 90 year-olds.

In the field of mathematics such a general latent ability that causes the performance on multiple related mathematical test is often (implicity) assumed. For example, when higher order latent variables are introduced to model individual differences (e.g., Aunola et al., 2004, Muthén et al., 1991).

A second, more recent, theory was proposed by van der Maas et al. (2006) as an alternative explanation for the positive manifold. In mutualism theory, development is seen as a complex system of (positively) interacting processes, where learning one process (skill) supports learning of the other processes in the system. The proposed mutualism model of general intelligence explains the positive manifold by positing mutually beneficial relations between the different abilities during development. The strength of these mutual relations between abilities are captured in the $\mathbf{M}$ matrix in the mutualism model and can be both unidirectional as for example in investment theory where growth in crystallized intelligence is fueled by niet growth in, maar juist 'level' fluid intelligence Cattell (1971) - or bidirectional. These bidirectional facilitating relations have previously been observed between the development of cognitive strategies and short-term memory (Siegler and Alibali, 2005), vocabulary and reading ability (Quinn et al., 2015) and between subjective and objective memory in aging (Snitz et al., 2015). In mathematics, van der Ven et al. (2012) found a positive interactions between changes in math skills and changes in working memory, suggesting mutual influences in their development.

Contrary to the $g$-factor theory, the mutualism theory predicts a strengthening of the correlational structure between performance on different cognitive tasks during development. Mutualism theory posits that all cognitive processes could initially be unrelated and then become related due to the positive interactions during development.

In this paper we compare both theoretical accounts of the positive manifold with respect to developing mathematical abilities. We investigate the relation between the development of simple (counting and addition) and more advanced (multiplication and division) mathematical abilities. Both sets of abilities are especially well suited for study because individual differences in these skills are often highly correlated () and ... hier mist iets For both sets of abilities, we examine two competing predictions that follow from each of the two developmental accounts: the cross-sectional correlational structure between domains and longitudinal coupling between domains over time.

First, we tested whether the $g$-factor or the mutualism theory best describes the development of the correlational structure between different math skills. Based on the idea of Gignac (2014), we tracked the development of the correlations between skills over time. In this bivariate approach the straightforward correlation coefficient contains all the necessary information to test whether the strength of the positive manifold is indeed stable or increases during development. Second, using latent change score model [ $^{2}$ (LCSM; McArdle, 2001, 2009, Ferrer and McArdle, 2010, Kievit et al., 2017a), we compared a set of models associated with the $g$-factor and mutualism accounts of the positive manifold in performance on different mathematics tests. To this end we analyzed data of a large group of children that frequently played different games in Math Garden.

To summarize, the goals of the current study are to examine whether $g$-theory or mutualism theory best explains the positive manifold in mathematical abilities. Mutualism theory predicts (strong) bidirectional relationships between different abilities during development whereas g-theory does not. Testing this prediction requires translating it into a testable statistical hypothesis that can be applied to longitudinal data. The next section provides these methods, as well as a description of the data.

\footnotetext{
${ }^{2}$ Sometimes referred to as latent difference score models.
} 


\section{Method}

\subsection{Modeling Framework: Latent change score models}

Latent change score models are structural equation models where the (latent) variables of interest are represented as change scores between time-points. The score on variable $y$ of person $p$ at time-point $t$ is regressed onto the score of $t-1$ as follows:

$$
y_{p t}=\beta_{t, t-1} y_{p t-1}+\Delta_{p t} .
$$

Setting the regression coefficient $\beta_{t, t-1}=1$ results in a $\Delta_{p t}$ that reflects the change in the scores between both time-points:

$$
\Delta_{p t}=y_{p t}-y_{p t-1}
$$

In the current paper we extend the LCSM to bivariate LCSM (McArdle, 2001) which allows us to investigate the relationship between the development of abilities in two separate domains as follows:

$$
\begin{aligned}
& \Delta_{1, p t}=\beta_{1} y_{1, p t-1}+\gamma_{21} y_{2, p t-1} \\
& \Delta_{2, p t}=\beta_{2} y_{2, p t-1}+\gamma_{12} y_{1, p t-1}
\end{aligned}
$$

In this model a change score is defined for both domains $(1,2)$. We use this framework to model growth using two different components. First, $\beta$, is a self-feedback parameter that relates the change between time-points to the scores on the previous time-point. A positive $\beta$ reflects accelerating growth and a negative $\beta$ indicates damping, regression to the mean, or ceiling effects. Second, and most important, are the coupling parameters $\gamma$. These reflect the effect of the previous score in one domain (i.e., at t-1) on the change in score in another domain. By examining the $\gamma_{21}$ and $\gamma_{12}$ parameters, we can determine which domains influence the development of other domains. Both $\gamma$ and $\beta$ parameters predict change scores and can therefore capture nonlinear processes (Ghisletta and De Ribaupierre, 2005).

We formulated a set of LSCM's that allow a direct comparison of two developmental theories by focusing on different ways to model change scores, which capture the amount of development between different time-points. We assumed that differences between $g$-factor and mutualism models (both with uni- and bidirectional effects) would show up in differences in the $\gamma$ parameters. This is because there is a clear relation between the $\gamma$ parameters in the LCSM and the $\mathbf{M}$ matrix in the mutualism model (effect of $\mathbf{M}$ is also defined on change scores; see van der Maas et al. (2006)). Using LCSM's we formulated different models based on hypothesis of either $g$-factor or different mutualistic accounts on the development of mathematical abilities, see Figure 1. For both models we included covariates of age (at T1) and amount of practice (number of items solved before T1) to account for possible differences between children in the baseline scores (T1). We did not include any covariates on other variables in the model because the developmental processes between the waves should be solely explained by the dynamics defined in the different LCSM's.

First, for the $g$-factor model, the latent $g$-factor is the underlying mechanism that steers development (i.e., change) in all domains. Hence, the change processes are defined at this latent level $\left(\Delta_{g 1}\right.$ and $\left.\Delta_{g 2}\right)$, and the observed scores and changes in both domains are expected to be caused by changes at the higher latent level. This results in a univariate LCSM, since only a single set of change factors at the level of $g$ is required. We imposed measurement invariance over time and allowed the observed scores within each domain to be correlated over time.

Second, for the mutualism model, we formulated a set of bivariate LCSM's with separate change scores for each domain and then successively added coupling parameters to connect the changes (development) across domains. For both the simple (addition and counting) and the advanced (multiplication and division) pairs of abilities, this resulted in four LCSM's: (1) a model without coupling parameters (see Appendix B for a description of the differences between this bivariate LCSM without coupling and the univariate LCSM, 

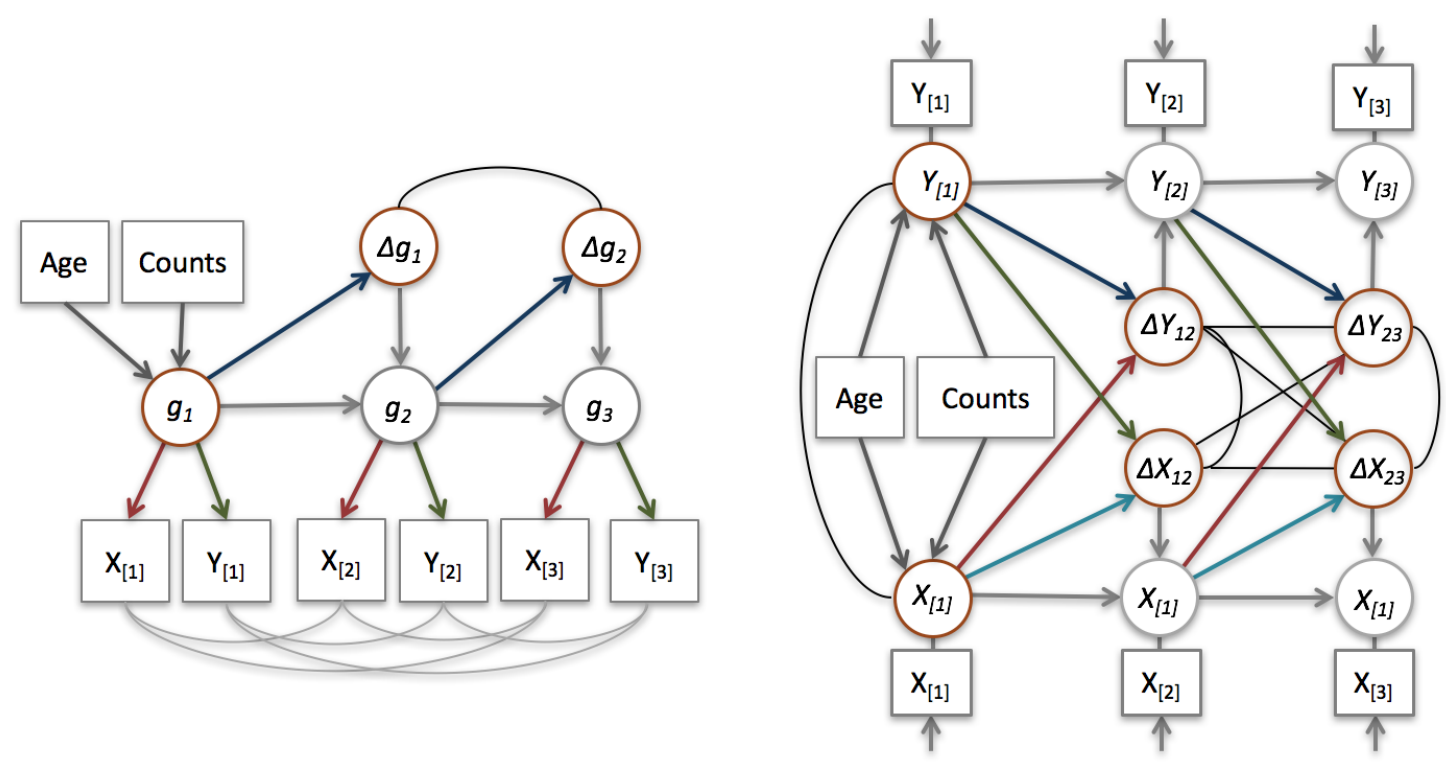

Figure 1: Specifications of the latent change score models of $g$-factor and mutualism accounts of cognitive development. Key parameters are color coded, and the same color indicates parameter equalities. Means and variances were estimated for all orange colored latent variables.

the $g$-factor model), (2\& 3$)$ two models with unidirectional coupling where only one of the two coupling parameters was present (for example an increase in counting results in growth in addition but not vice versa) or (4) a model with bidirectional coupling parameters (mutualism), where changes in each domain influences development in the other domain. The no-coupling model resembles the correlated growth model of Quinn et al. (2015) and functions as a baseline model which we test against to determine the presence of significant coupling parameters. Also, as indicated by a simulation study (see Appendix B), if the coupling parameters are set to zero the model fit of the no-coupling model resembles the model fit of the $g$-factor model, which further supports its use as a baseline model.

In the bivariate LCSM's the (residual) change factors $\left(\Delta X_{1}, \Delta X_{2}, \Delta Y_{1}\right.$ and $\left.\Delta X_{2}\right)$ are allowed to be correlated. These correlations imply that after possible coupling effects, the change factors of different variables and at different time-points are related. These correlations, or 'structured residuals' (cf. Curran et al., 2014) reflect the possible effects of: (1) a possible larger set of variables that influence growth in both measured domains, which are not included in the analyzed data set (e.g., working memory, processing speed, reasoning) and/or (2) a mismatch between measurement density during data collection and the time steps with which natural development unfolds (see Appendix B). Both of these mechanisms may be present in our sample.

Both models are formulated based on a data set with three time-points. In constructing a data set based on data from an online learning systems there is a trade-off between either a large number of time-points with fewer children or a smaller number of time-points resulting in a large number of participants. We aimed for the latter and selected three waves. This allows us to test the competing models with sufficient precision, and even allows for extensions such as incorporating the estimation of correlations between changes between different time-point and within domains, whilst retaining sufficiently large sample sizes of children who played regularly. Also, the formulation of both models including the correlational structure on the change scores is straightforward, and no higher order change-factors can or have to be specified as for example in Ferrer and McArdle (2003, 2004). Moreover, sampling three full school years allows us to study a sufficient period of learning and development. 


\section{$2.2 \quad$ Instruments}

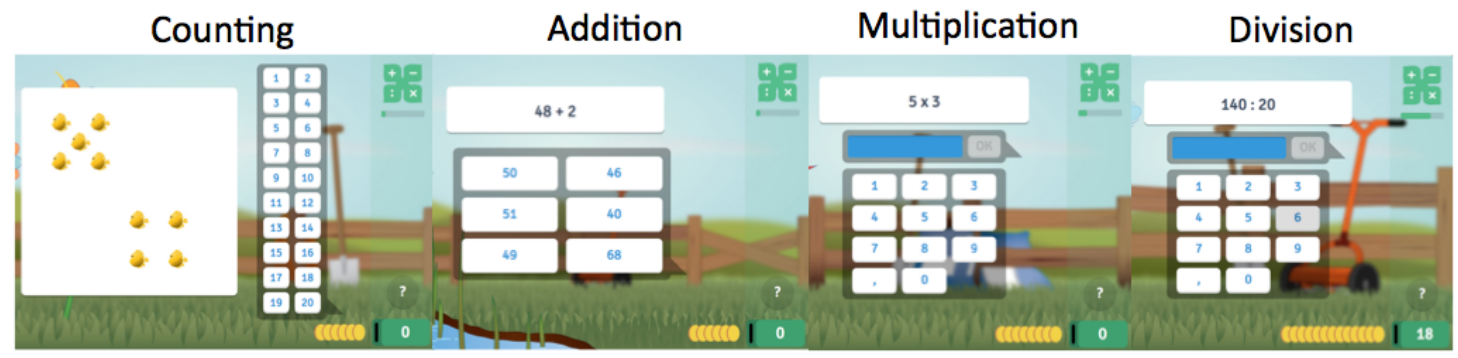

Figure 2: Screenshots of the counting, addition, multiplication and division games in the Math Garden online learning platform. On counting and addition tasks children click an answer option to give a response. For multiplication and division children use the numeric keypad to supply their solution. The coins at the bottom disappear gradually, one per second, and represent how the games are scored - one point for each remaining coin after supplying the correct solution.

Data was collected using a popular Dutch online adaptive practice system for mathematics (Math Garden; Straatemeier, 2014, Klinkenberg et al., 2011; Brinkhuis et al., 2018). The system consists of multiple games that measure different mathematical abilities. In each game a player's ability is re-estimated after every response using a variant of the Elo-algorithm (Elo, 1978, Klinkenberg et al., 2011), which takes both accuracy and response time into account (Maris and Van der Maas, 2012). For more details on the Math Garden and its psychometric properties, see Appendix A. In this study we focus on two basic skills: counting (Jansen et al. 2014) and addition, and two more advanced skills: multiplication (van der Ven et al., 2015, Hofman et al. 2017) and division. Each skill is measured by a separate game. Figure 2 shows a screen shot of an example item of each game. The estimated ability scores (comparable to factor scores in structural equation models, see Appendix A were used as input variables in the LCSM's.

Children (or their parents) who indicated that they did not want to participate in scientific research conducted with Math Garden were excluded from the analyses. This research study was approved by the psychology department's Ethics Committee.

\subsubsection{Data Selection: Tracking Development}

We selected the ability estimates of children who played at least thirty items in one week between the start of the school year in 2013 and the end of the school year in 2016 (covering three full school years). For the investigation of the development of counting and addition we selected children in first grade, born in 2007 ( $M=7.7, S D=1.2$ years), at the start of the data collection (around nine years old at the end of data collection - end of 3rd grade). For the investigation of multiplication and division development we selected children born in 2006 ( $M=9.7, S D=1.3$ years) who were on average eight years old at the start of the data collection (beginning of 2nd grade). By selecting children based on birth cohorts we ensured that the childrens' ages increased (linearly) over time, although children could enter and the leave the data collection at different time-points by deciding to start or quit playing a particular game. The same data selection approach was used by Brinkhuis et al. (2015). We plotted the number of selected children for each week in the upper panel of Figure 3 School holidays are denoted by the grey areas. For the analyses on the changes in correlations between the math abilites we only included weeks in which a least 25 children played both games.

\subsubsection{Data Selection: Latent Change Score Models}

For the estimation of the LSCM's we constructed a data set with three time-points. We selected the ability estimates of children for the three separate years in the first month (2013-09-02 - 2013-10-04; 2014-09-01 - 
2014-10-03; 2015-08-31 - 2015-10-02), the middle (2014-01-06 - 2014-02-07; 2015-01-05 - 2015-02-06; 2016-01-04 - 2016-02-05) and at the end of the school year (2014-05-12 - 2014-06-27; 2015-05-11 - 2015-06-26; 2016-05-09 - 2016-06-24) giving us nine ability estimates per math task. We defined these time-points based on school periods to capture our hypothesis that the amount of schooling is the dominant factor in driving developmental change. In addition, only data of children in grades one to six, between four and twelve years old 3 and who solved at least thirty items on the two domains within one or multiple periods were selected. For the analysis based on the counting and addition games we only selected children under ten years old given that the counting game is most relevant for this age group.

All scores were standardized based on the mean score and $S D$ at T1. The final data sets and code for all analyses and simulations are available online $4^{4}$

\subsection{Model Estimation and Comparison}

LCSM's were estimated in Lavaan (Rosseel, 2012) using Full Information Maximum Likelihood with robust standard errors to account for missings and non-normality. To assess the overall model fit we used the following tests with guidelines based on Schermelleh-Engel et al. (2003); chi-square test, the CFI (acceptable fit $.95-.97$, good fit $>0.97$ ), the RMSEA (acceptable fit $<0.08$, good fit $<0.05$ ), the SRMR (acceptable fit $.05-.10$, good fit $<0.05)$. We compared the model fit using information criteria (AIC and BIC) and Akaike Weights (Wagenmakers and Farrell, 2004). These weights express the evidence for each model given the observed data and the set of candidate models.

\section{Results}

\subsection{Developing Correlations}

First, we investigate the development of the correlations between the two math skills in each data set. The upper-panel plots of Figure 3 show the number of participants for each week; the green line indicates the number of participants that played both games. The number of participants varied from 25 (minimum) to 250 at the peaks. The division game was the least popular game, with about 100 participants during peak periods. Not surprisingly, in each math domain the average rating clearly increases during the three years of data collection (middle-panel plots), with the exception of the summer holidays where a dip is observed. The lower-panel plots show the correlation between the two math domains for each week (only weeks where $¿ 25$ children played both games). The grey areas indicate the $95 \%$ confidence interval and the blue dashed line shows the predicted values from a weighted linear regression model where the correlation is predicted by time (week number) using the number of observations per week as weights. For both data sets this regression coefficient was positive and significant (1 counting and addition: $\beta=.00094, t(107)=4.779, p<.001$, $R^{2}=.166 ; 2$ multiplication and division: $\left.\beta=.00087, t(100)=4.458, p<.001, R^{2}=.158\right)^{5}$. This clearly indicates that the estimated correlation coefficients increased over time.

This analysis provides indirect evidence that mutualistic interactions drive the development of these math skills, resulting in increased correlations over time. This evidence, however, is inconclusive since the change could be caused by either changing error variances in both scores or changes in the strength of the factor (Gignac, 2014; Molenaar et al. 2010). Yet the current result naturally follows from a mutualism perspective, while it is more difficult to reconcile from a $g$-factor perspective. In the next analyses we provide a more direct comparison of both theoretical accounts explaining the positive manifold, using a set of LCSM's.

\footnotetext{
${ }^{3}$ We performed a reliability check on the information about age and grade, where children who deviated more than two standard deviations from the average grade per class were deleted

${ }^{4}$ See: https://osf.io/gcdw3/

${ }^{5}$ Since some cases might have overlapped for different data-points we used a bootstrap method and performed a permutated null-hypothesis test. Using 50.000 replications, none of the sampled t-statistics exceeded the observed t-statistics, supporting the significant results of the presented tests for both data sets
} 

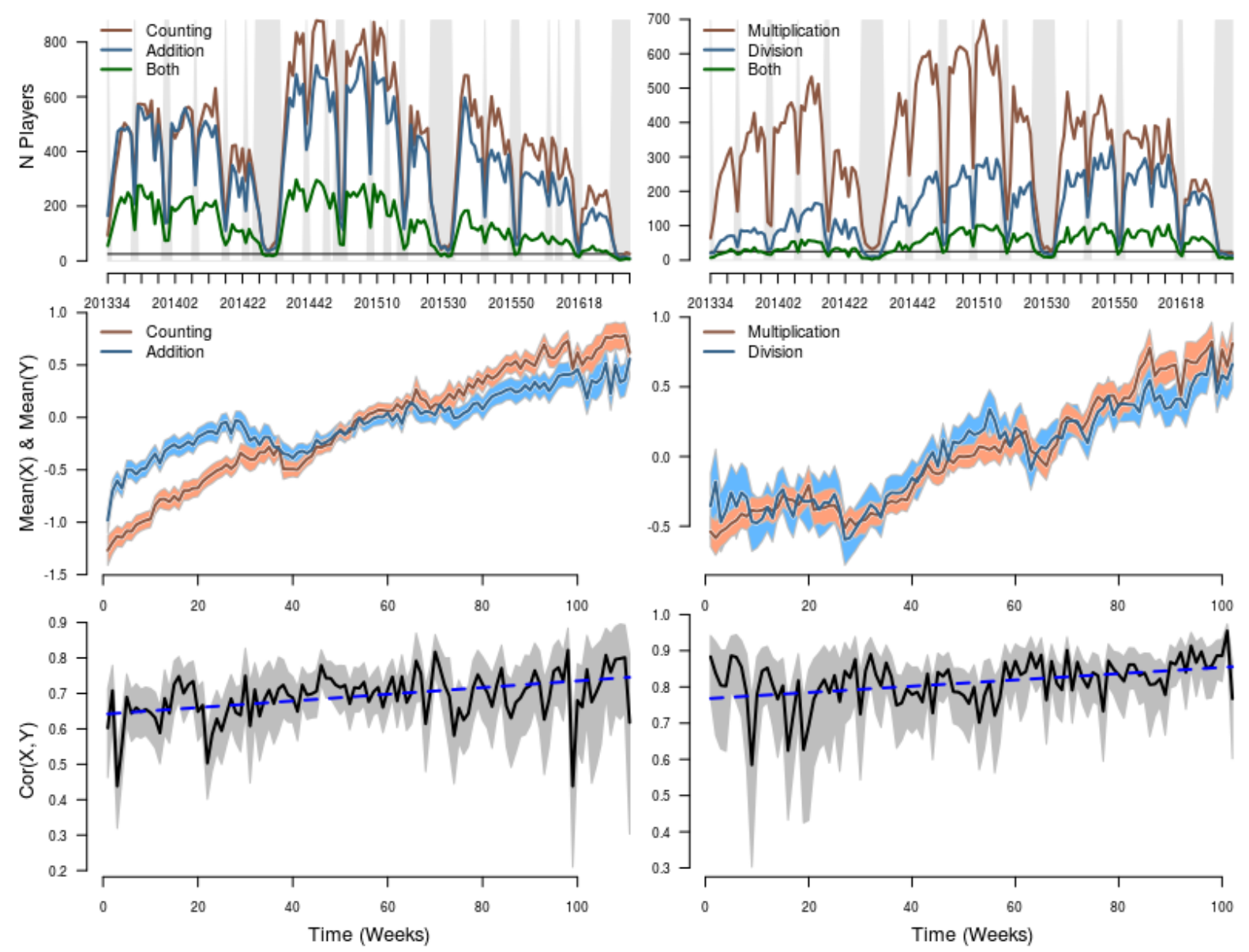

Figure 3: The number of selected players in our dataset for each game (upper-panels; gray areas indicate school holidays), the mean ability scores for each game (middle-panels; with the $95 \%$ confidence interval) and the correlations between scores for children in a specific birth cohort (children born in 2007 and 2006, respectively). The gray area indicates the $95 \%$ confidence interval and the dashed blue line is the regression line which indicates a significant increase in both correlations over time.

\subsection{Latent Change Score Models}

The data selection resulted in 11,980 participants for the counting vs addition data set and 12,368 participants for the multiplication vs division data set (of which 697 and 1,054 cases respectively contained data on both variables at all three time-points). 218 participants from the counting vs addition data set and 8 participants from the multiplication vs division data set were deleted due to outliers (ability score that deviated more than three $S D s$ from the mean). Table 1 shows the total number of participants included at each time-point, the mean and SD of the unstandardized ratings, and the correlations between the ability scores at each time-point for each data set.

We fitted the five different LCSM's on both data sets. Table 2 shows the different fit indices for each of the models. For both data sets, all models showed a good fit as indicated by the CFI, RMSEA and SRMR, with only small differences between these three fit indices. The AIC and BIC, which take both fit and model complexity into account, showed that the four bivariate LCSM's (a no-coupling, two unidirectional coupling and a mutualism model with bidirectional coupling) fit better than the $g$-factor model. Furthermore, 
Table 1: Descriptives for Counting and Addition data set (top section) and Multiplication and Division data set (bottom section).

\begin{tabular}{llllllll}
\hline Domains & Variable & Count T1 & Count T2 & Count T3 & Add T1 & Add T1 & Add T1 \\
\hline Counting & Mean & -5.040 & -4.627 & -4.482 & -9.513 & -8.451 & -7.638 \\
$\&$ & SD & 1.353 & 1.325 & 1.375 & 3.410 & 3.389 & 3.328 \\
Addition & Count T1 & 6279 & 3412 & 2672 & 4561 & 3926 & 3222 \\
& Count T2 & 0.795 & 7580 & 3952 & 3644 & 5549 & 4505 \\
& Count T3 & 0.712 & 0.772 & 6498 & 2838 & 4253 & 4731 \\
& Add T1 & 0.782 & 0.706 & 0.654 & 6106 & 3581 & 2958 \\
& Add T2 & 0.764 & 0.778 & 0.709 & 0.881 & 7862 & 4356 \\
& Add T3 & 0.726 & 0.737 & 0.759 & 0.835 & 0.894 & 7029 \\
\hline Multipli- & Mean & -9.892 & -8.370 & -7.067 & -12.220 & -11.156 & -9.966 \\
cation & SD & 3.798 & 4.215 & 4.495 & 5.923 & 6.374 & 6.738 \\
\& & Mult T1 & 7104 & 4583 & 3649 & 4254 & 4842 & 3986 \\
Division & Mult T2 & 0.887 & 8996 & 5474 & 3679 & 6541 & 5969 \\
& Mult T3 & 0.810 & 0.899 & 7613 & 2825 & 4999 & 6232 \\
& Div T1 & 0.855 & 0.817 & 0.757 & 5325 & 3541 & 2779 \\
& Div T2 & 0.817 & 0.869 & 0.830 & 0.897 & 8386 & 4991 \\
& Div T3 & 0.773 & 0.827 & 0.873 & 0.834 & 0.917 & 8156 \\
\hline
\end{tabular}

Note. The lower-diagonal values represent the correlations between the scores on each variable. The upper-diagonal values represent the number of participants with scores on a single (diagonal) or set of variables (off-diagonal). $\mathrm{T}=$ time-point , Count $=$ Counting, Add $=$ Addition, Mult $=$ Multiplication, Div $=$ Division

Table 2: Fit statistics for the estimated Latent Change Score models for Counting and Addition (top section) and Multiplication and Division (bottom section).

\begin{tabular}{lllllllll}
\hline Domains & Model & Chi & df & CFI & RMSEA & SRMR & AIC & BIC \\
\hline Counting & -Factor & 571.66 & 15 & 0.987 & 0.056 & 0.046 & 142138 & 142352 \\
$\&$ & Mutualism & 461.48 & 11 & 0.989 & 0.059 & 0.038 & 142036 & 142279 \\
Addition & Uni. $(\mathrm{C} \rightarrow \mathrm{A})$ & 475.05 & 12 & 0.989 & 0.057 & 0.039 & 142048 & 142284 \\
& Uni. $(\mathrm{C} \leftarrow \mathrm{A})$ & 470.95 & 12 & 0.989 & 0.057 & 0.038 & 142044 & 142279 \\
& No coupling & 485.24 & 13 & 0.989 & 0.056 & 0.038 & 142056 & 142284 \\
\hline Multipli- & g-Factor & 671.52 & 15 & 0.989 & 0.059 & 0.030 & 149304 & 149520 \\
cation & Mutualism & 517.17 & 11 & 0.991 & 0.061 & 0.026 & 149158 & 149403 \\
$\&$ & Uni. $(\mathrm{M} \rightarrow \mathrm{D})$ & 575.44 & 12 & 0.990 & 0.062 & 0.027 & 149214 & 149452 \\
Division & Uni.(M $\leftarrow \mathrm{D})$ & 521.91 & 12 & 0.991 & 0.059 & 0.026 & 149161 & 149398 \\
& No coupling & 597.61 & 13 & 0.990 & 0.060 & 0.026 & 149234 & 149464 \\
\hline
\end{tabular}

Note. The the best fitting models are printed in bold. Uni. = Unidirectional Model

according to the AIC the mutualism model including two sets of coupling parameters showed a better fit than both unidirectional models and the bivariate model without coupling. Using AIC-weights (Wagenmakers and Farrell, 2004), we can represent the conditional probability of each model, given the observed data, within our set of candidate models.

Figure 4 shows these weights for each model and clearly indicates that the mutualism model outperforms all other candidate models in the counting vs addition data set. This differences is less prominent for multiplication vs division data set. For this data set the fit of the mutualism model only slightly outperforms the unidirectional model including a coupling parameter from division to multiplication. According to the BIC this unidirectional model provides a better fit, while comparison of the likelihoods indicates significantly better fit for the mutualism model $\left(X^{2}(1)=4.746, p=.029\right)$. Both models clearly outperform the $g$-factor 
Counting vs Addition

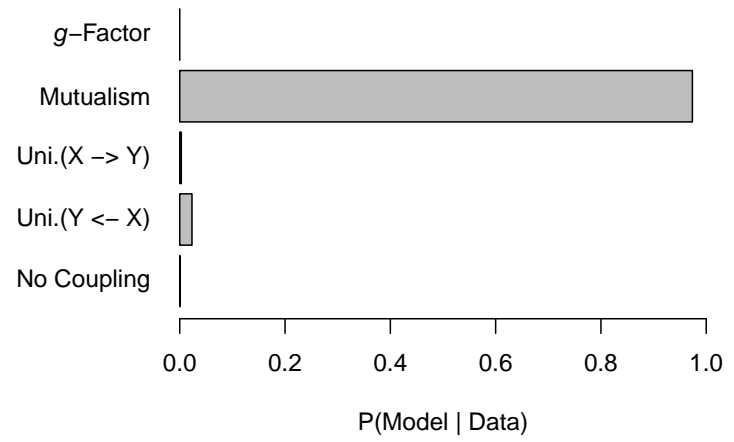

Multiplication vs Division

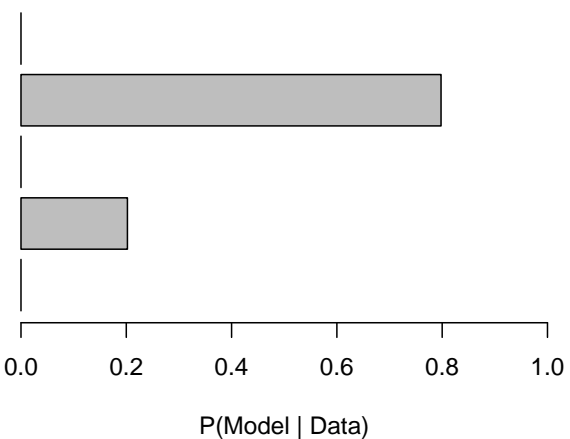

Figure 4: The AIC-weights of all models for both data sets, indicating the evidence for each model given the observed data and the set of candidate models.

account. Although the evidence of bidirectional coupling is less clear, the mutualism model provides the best description of the data $\sqrt{6}$

In the following section we investigate the parameter estimates of the mutualism model in more detail. Figure 5 shows all parameter estimates for the mutualism model fitted to the counting and addition data set. Since the model parameters for the counting and addition data set were very similar to those of the multiplication and division data set, we first describe the general model details and then go on to describe the main differences between the results for the two data sets (see Appendix C for all model parameters of the multiplication and division data set).

Both age and the number of items solved before T1 (counts) have large effects on the scores at T1, and the corrected scores in both domains are, as expected, still highly correlated. The means of the change scores indicate significant positive changes in ability from one time-point to the next. The self-feedback parameters are negative, which indicates that participants with high scores have lower change scores than participants with lower scores. These negative effects are often found, both in longitudinal data (e.g., Kievit et al., 2017b) and in dynamic testing (e.g., Stevenson et al., 2013). This could indicate regression toward the mean, as well as reflecting a deceleration in mathematical development over time. In principle these negative effects can also be caused by ceiling effects, but these were not present in the data. More importantly, the coupling parameters are positive, indicating that high scores in one domain result in more change in the other domain, as is predicted by the mutualism model. The self-feedback and coupling parameters explained $7.8 \%$ and $8.4 \%$ of the variance respectively for the first and second change score of addition (X) and $3.6 \%$ and $4.3 \%$ respectively for the first and second change score of counting $(\mathrm{Y})$. For the multiplication and division data set these explained variances were $3.1 \%$ and $2.6 \%$ for the first and second change score of multiplication (X) and $1.9 \%$ and $0.4 \%$ for first and second change score of division $(\mathrm{Y})$. Although these values differ, in general they indicate that a non-trivial part of the variance in the change scores can be explained by the effects included in the model.

We found significant correlations between the latent change scores of the different domains at the same time-points (respectively, .546 and .566 for the first and second change scores). These correlations show that after including the coupling parameters the residual changes are still positively correlated. This suggests that, unsurprisingly, it is likely that other cognitive or developmental factors influence change rates in both

\footnotetext{
${ }^{6}$ For the multiplication vs division data set, the results were comparable if no covariates were included in the model and the mutualism model clearly outperformed the other models. For the counting vs addition data set this was also the case when only Age was included as a covariate. Excluding Age resulted in a small variance in one of the change factors (no difference from zero). This suggested that no individual differences were present in this change-score. This caused problems for the estimation of the covariance matrix of the change scores, resulting in unreliable model comparison.
} 


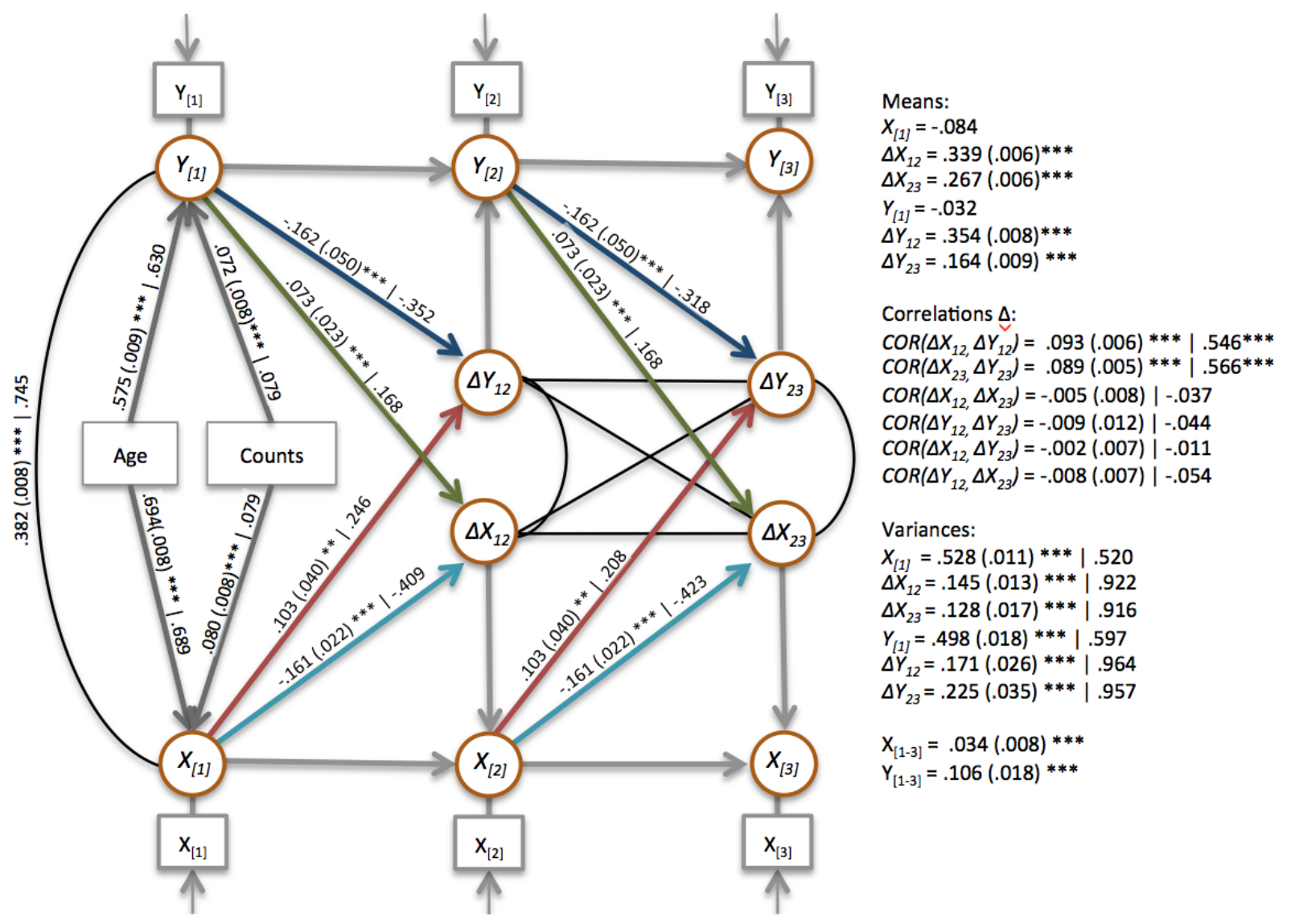

Figure 5: The estimated model parameters for the best fitting mutualism model for the counting and addition data set. The first value denotes the unstandardized coefficient, with the standard error between brackets, and the value after the vertical bar denotes the standardized coefficient. The stars indicate the significant levels $\left(*=\mathrm{p}<.05,{ }^{* *}=\mathrm{p}<0.01\right.$ and $\left.{ }^{* * *}=\mathrm{p}<.001\right)$. The grey lines indicate fixed parameters. The observed scores (X and $\mathrm{Y}$ ) are the latent trait estimates provided by Math Garden.

domains (see Appendix B or that the analyzed data does not include enough waves to perfectly describe the true developmental processes (see Appendix C). Possible other cognitive candidates include growth of working memory, processing speed and attention factors. For the multiplication vs division data set these correlations were respectively .638 and .657 . The remaining correlations between the change scores (correlations between $\Delta X_{12}$ and $\Delta X_{23}, \Delta Y_{12}$ and $\Delta Y_{23}, \Delta X_{12}$ and $\Delta Y_{23}$ and between $\Delta Y_{12}$ and $\Delta X_{23}$ ) did not differ from zero in the counting vs addition data set. In the multiplication vs division data set these correlations were non-zero, varying between .104 and .199. The remaining residual correlations between the change factors $\Delta_{1, t p}$ and $\Delta_{2, t p}$, after including the direct links between learning processes, could be caused by unobserved coupling effects (see Appendix B), but also allows for an additional $g$-factor effect. Hence, these results are inconclusive about the explanation of the remaining correlations structure of the change factors.

To conclude, consistent with the conclusion from the analyses of the developing correlations, the comparison of different LCSM's indicated that, in line with the mutualism model, bidirectional coupling is present between the development of counting and addition, as well as the development of multiplication and division. A final alternative account to explain the large positive correlational structure between change scores can again posit a $g$-factor to explain these correlations between change-scores. Hence these results indicate that a non-trivial proportion of developmental processes can be ascribed to mutualistic processes, but a considerable proportion of the shared variance remains to be explain, either by a $g$-factor account or by incorporating 
more dynamically coupled cognitive domains.

\section{Discussion}

We compared two leading theories on the development of intelligence, $g$-factor and mutualism, using a large longitudinal dataset of primary school children's developing mathematics skills. The main finding is that the $g$-factor account of mathematics learning provides a too simplistic account of the developmental processes. Instead, to understand how children learn mathematics, a dynamic mutualism account, where learning one skill benefits learning another, and vice versa, needs to be added to the equation.

We found that learning to count and add positively influence each others development. Similarly, multiplication and division skills grow and benefit from each other over time. There are two mechanisms that explain these mutually beneficial interactions (i.e., coupling) Kievit et al. (2017b). On the one hand, children may transfer skills from one domain to another. One example is that certain counting skills may be needed to solve specific addition problems and, on the other hand, some counting tasks are easier to solve using addition strategies (Carpenter and Moser, 1984, counting sets of elements). Another example, is that insights gained in division facilitate learning multiplication, for example understanding the inverse relation between division and multiplication (Robinson and Dubé, 2009). On the other hand, an indirect mechanism is also possible, especially since transfer of skills from one task to another are usually weak (Barnett and Ceci, 2002). For example, a teacher observes that a child is really good at multiplication and provides him/her with challenging division tasks sooner than other children. In this case, high ability children in one domain have the chance to improve their abilities in another domain, but low ability children do not. Such selection effects are common in education (e.g., (Verachtert et al. 2010)) and would also lead to coupling between the two domains where the school environment serves as a mediator (see for example the proposed mutualism model of intelligence by $\operatorname{Kan}$ (p. 1012012 ) where both direct and indirect environmental effects are included).

Although we analyzed 'big' longitudinal data collected in a natural learning setting, the sample has some shortcomings. First, the children selected the games they wanted to play. Most likely higher-ability children chose to play more difficult games earlier than lower-ability children; this would mean that we compared the scores of higher-ability children at earlier time-points with those of lower-ability children at later time-points. This self-selection bias would likely underestimate the average level of change between time-points. However, since this selection mechanism likely underestimates rather than overestimates average changes in ability, our findings remain valid. Second, the the density of data collection determines the level of detail we see in the window of development, which of course should mimic (to a reasonable extent) real developmental processes. But, coupling effects that occur on a different time-scale than the detail of data collection could influence the correlational structure of the change scores. The time-points we used, three within a full school year, probably resulted in an underestimation of the true coupling effects. This is also supported by the high correlations between the change factor of different skills at the same time-points. Third, as with other longitudinal data analyses, practice effects could have influenced our results (Salthouse and Tucker-Drob, 2008). However, as explained by Kievit et al. (2017b), the presence of practice effects would only underestimate mutualistic coupling effects, because ability changes caused by practice effects would be less likely to result in coupling with other skills. Fourth, the estimated ability scores are based on responses in a low-stakes testing environment and children might be distracted or not motivated to do their best. However, we do not expect that this caused biases in the data for two reasons. First, we analyzed a large group of children, which means that possible motivational differences between children are likely to cancel each other out. Second, the motivational effects probably did not differ between time-points.

The model we used assumes that coupling between math skills is the same for all children. However, it is likely that children differ in the degree to which different skills influence each others growth and should be examined in future research. van der Maas et al. (2006) showed that such individual differences in coupling between domains leads to vastly different learning curves, and also the complex factor structure often found in real data. For example, Ferrer et al. (2010) found that developing readers and children with reading difficulties (compensated and poor readers) differed in how IQ and reading ability were linked over time. Individual differences in coupling between domains could provide a new window for understanding development, for 
example for early detection of abnormal developmental trajectories.

Our findings have important implications for understanding the processes involved in the development of mathematics abilities. The mutualistic effects found in this study imply that during development different skills become more intertwined and possibly, at some point, become so strongly connected that they can be seen as a higher-level unidimensional skill. Hence, these higher-level factors emerge from direct links during development. These mutualistic effects imply accelerated growth during the key developmental period and differences in learning speed would be determined by the strength and number of intertwined skills. If our results replicate, future research should study large-scale multivariate longitudinal data of important skills during development to get a more complete picture of co-developing processes involved in cognitive development, and mathematics learning in particular. 


\section{References}

Anderson, M., 2017. Binet's error: Developmental change and individual differences in intelligence are related to different mechanisms. Journal of Intelligence 5 (2), 24.

Aunola, K., Leskinen, E., Lerkkanen, M.-K., Nurmi, J.-E., 2004. Developmental dynamics of math performance from preschool to grade 2. Journal of Educational Psychology 96 (4), 699.

Barnett, S. M., Ceci, S. J., 2002. When and where do we apply what we learn?: A taxonomy for far transfer. Psychological bulletin 128 (4), 612 .

Bartholomew, D. J., Deary, I. J., Lawn, M., 2009. The origin of factor scores: Spearman, thomson and bartlett. British Journal of Mathematical and Statistical Psychology 62 (3), 569-582. URL http://dx.doi .org/10.1348/000711008X365676

Borsboom, D., Mellenbergh, G. J., Van Heerden, J., 2003. The theoretical status of latent variables. Psychological review 110 (2), 203.

Brinkhuis, M. J., Bakker, M., Maris, G., 2015. Filtering data for detecting differential development. Journal of Educational Measurement 52 (3), 319-338.

Brinkhuis, M. J., Maris, G., 2009. Dynamic parameter estimation in student monitoring systems. Measurement and Research Department Reports (Rep. No. 2009-1). Arnhem: Cito.

Brinkhuis, M. J. S., Savi, A. O., Hofman, A. D., Coomans, F., van der Maas, H. L. J., Maris, G., 2018. Learning as it happens: A decade of analyzing and shaping a large-scale online learning system. Retrieved from PsyArXiv, 10.17605/OSF.IO/G4Z85 - 1 .

Carpenter, T. P., Moser, J. M., 1984. The acquisition of addition and subtraction concepts in grades one through three. Journal for research in Mathematics Education, 179-202.

Cattell, R. B., 1971. Abilities: Their structure, growth and action. Houghton-Mifflin, Boston.

Curran, P. J., Howard, A. L., Bainter, S. A., Lane, S. T., McGinley, J. S., 2014. The separation of betweenperson and within-person components of individual change over time: a latent curve model with structured residuals. Journal of consulting and clinical psychology $82(5), 879$.

Elo, A. E., 1978. The rating of chessplayers, past and present. Arco Pub.

Epskamp, S., Cramer, A. O., Waldorp, L. J., Schmittmann, V. D., Borsboom, D., et al., 2012. qgraph: Network visualizations of relationships in psychometric data. Journal of Statistical Software 48 (4), 1-18.

Ferrer, E., McArdle, J., 2003. Alternative structural models for multivariate longitudinal data analysis. Structural Equation Modeling 10 (4), 493-524.

Ferrer, E., McArdle, J. J., 2004. An experimental analysis of dynamic hypotheses about cognitive abilities and achievement from childhood to early adulthood. Developmental psychology 40 (6), 935-951.

Ferrer, E., McArdle, J. J., 2010. Longitudinal modeling of developmental changes in psychological research. Current Directions in Psychological Science 19 (3), 149-154.

Ferrer, E., Shaywitz, B. A., Holahan, J. M., Marchione, K., Shaywitz, S. E., 2010. Uncoupling of reading and iq over time. Psychological Science 21 (1), 93-101, pMID: 20424029.

URL http://dx.doi .org/10.1177/0956797609354084

Geary, D. C., Brown, S. C., Samaranayake, V., 1991. Cognitive addition: A short longitudinal study of strategy choice and speed-of-processing differences in normal and mathematically disabled children. Developmental psychology 27 (5), 787. 
Geary, D. C., Hoard, M. K., Byrd-Craven, J., DeSoto, M. C., 2004. Strategy choices in simple and complex addition: Contributions of working memory and counting knowledge for children with mathematical disability. Journal of Experimental Child Psychology 88 (2), 121 - 151.

URL http://www.sciencedirect.com/science/article/pii/S0022096504000335

Ghisletta, P., De Ribaupierre, A., 2005. A dynamic investigation of cognitive dedifferentiation with control for retest: evidence from the swiss interdisciplinary longitudinal study on the oldest old. Psychology and aging 20 (4), 671.

Gignac, G. E., 2014. Dynamic mutualism versus g factor theory: An empirical test. Intelligence 42, 89-97.

Halberda, J., Mazzocco, M. M., Feigenson, L., 2008. Individual differences in non-verbal number acuity correlate with maths achievement. Nature 455 (7213), 665-668.

Hofman, A. D., Visser, I., Jansen, B. R. J., Marsman, M., van der Maas, H. L. J., 2017. Fast and slow strategies in multiplication. Retrieved from PsyArXiv, 10.17605/OSF.IO/AW3QQ - 1.

Jansen, B. R., Hofman, A. D., Savi, A., Visser, I., van der Maas, H. L., 2016. Self-adapting the success rate when practicing math. Learning and Individual Differences 51, 1-10.

Jansen, B. R., Hofman, A. D., Straatemeier, M., Bers, B. M., Raijmakers, M. E., van der Maas, H. L., 2014. The role of pattern recognition in children's exact enumeration of small numbers. British Journal of Developmental Psychology 32 (2), 178-194.

Jensen, A. R., 1998. The g factor: The science of mental ability. Westport CT USA: Praeger Publishers / Greenwood Publishing Group Inc.

Kan, K.-J., 2012. The nature of nurture: the role of gene-environment interplay in the development of intelligence. Ph.D. thesis, University of Amsterdam.

URL http://hdl.handle.net/11245/1.392628

Kievit, R., Brandmaier, A., Ziegler, G., van Harmelen, A.-L., de Mooij, S., Moutoussis, M., Goodyer, I., Bullmore, E., Jones, P., Fonagy, P., Consortium, N., Lindenberger, U., Dolan, R., 2017a. Developmental cognitive neuroscience using latent change score models: A tutorial and applications. bioRxiv.

Kievit, R. A., Lindenberger, U., Goodyer, I. M., Jones, P. B., Fonagy, P., Bullmore, E. T., Dolan, R. J., 2017b. Mutualistic coupling between vocabulary and reasoning supports cognitive development during late adolescence and early adulthood. Psychological Science, 10.1177/0956797617710785.

Klinkenberg, S., Straatemeier, M., van der Maas, H. L., 2011. Computer adaptive practice of maths ability using a new item response model for on the fly ability and difficulty estimation. Computers \& Education 57 (2), 1813-1824.

Kruis, J., Maris, G., 2016. Three representations of the ising model. Scientific Reports 6, 1-11.

Maris, G., Van der Maas, H., 2012. Speed-accuracy response models: Scoring rules based on response time and accuracy. Psychometrika 77, 615-633.

McArdle, J. J., 2001. A latent difference score approach to longitudinal dynamic structural analyses. In: R., C., duToit S., Sörbom, D. (Eds.), Structural equation modeling: Present and future. Scientific Software International, Lincolnwood, pp. 342-380.

McArdle, J. J., 2009. Latent variable modeling of differences and changes with longitudinal data. Annual review of psychology $60,577-605$.

McArdle, J. J., Ferrer-Caja, E., Hamagami, F., Woodcock, R. W., 2002. Comparative longitudinal structural analyses of the growth and decline of multiple intellectual abilities over the life span. Developmental psychology 38 (1), 115. 
Molenaar, D., Dolan, C. V., Wicherts, J. M., van der Maas, H. L., 2010. Modeling differentiation of cognitive abilities within the higher-order factor model using moderated factor analysis. Intelligence 38 (6), 611-624.

Molenaar, P. C., 2004. A manifesto on psychology as idiographic science: Bringing the person back into scientific psychology, this time forever. Measurement 2 (4), 201-218.

Murnane, R. J., John, B. W., Levy, F., 1995. The growing importance of cognitive skills in wage determination. The Review of Economics and Statistics 77 (2), 251-266.

Muthén, B. O., Kao, C.-F., Burstein, L., 1991. Instructionally sensitive psychometrics: Application of a new irt-based detection technique to mathematics achievement test items. Journal of Educational Measurement 28 (1), 1-22.

URL http://dx.doi.org/10.1111/j.1745-3984.1991.tb00340.x

Pelánek, R., 2016. Applications of the elo rating system in adaptive educational systems. Computers \& Education 98, 169-179.

Quinn, J. M., Wagner, R. K., Petscher, Y., Lopez, D., 2015. Developmental relations between vocabulary knowledge and reading comprehension: A latent change score modeling study. Child development 86 (1), $159-175$.

Robinson, K. M., Dubé, A. K., 2009. Children's understanding of the inverse relation between multiplication and division. Cognitive Development 24 (3), 310 - 321.

URL http://www.sciencedirect.com/science/article/pii/S0885201408000889

Rosseel, Y., 2012. lavaan: An R package for structural equation modeling. Journal of Statistical Software 48, $1-36$.

URL http://www.jstatsoft.org/v48/i02/

Salthouse, T. A., Tucker-Drob, E. M., 2008. Implications of short-term retest effects for the interpretation of longitudinal change. Neuropsychology 22 (6), 800.

Schermelleh-Engel, K., Moosbrugger, H., Müller, H., 2003. Evaluating the fit of structural equation models: Tests of significance and descriptive goodness-of-fit measures. Methods of psychological research online 8 (2), 23-74.

Siegler, R. S., Alibali, M. W., 2005. Children's thinking (4th ed.). Upper Saddle River, NJ: Prentice Hall.

Siegler, R. S., Duncan, G. J., Davis-Kean, P. E., Duckworth, K., Claessens, A., Engel, M., Susperreguy, M. I., Chen, M., 2012. Early predictors of high school mathematics achievement. Psychological science 23 (7), 691-697.

Siegler, R. S., Lortie-Forgues, H., 2014. An integrative theory of numerical development. Child Development Perspectives 8 (3), 144-150.

Snitz, B. E., Small, B. J., Wang, T., Chang, C.-C. H., Hughes, T. F., Ganguli, M., 2015. Do subjective memory complaints lead or follow objective cognitive change? a five-year population study of temporal influence. Journal of the International Neuropsychological Society 21 (9), 732-742.

Spearman, C., 1927. The abilities of man. Macmillan.

Stevenson, C. E., Hickendorff, M., Resing, W. C., Heiser, W. J., de Boeck, P. A., 2013. Explanatory item response modeling of children's change on a dynamic test of analogical reasoning. Intelligence 41 (3), $157-168$.

Straatemeier, M., 2014. Math Garden: A new educational and scientific instrument. Ph.D. thesis, University of Amsterdam. URL http://hdl.handle.net/11245/1.417091 
Tucker-Drob, E. M., 2009. Differentiation of cognitive abilities across the life span. Developmental psychology $45(4), 1097$.

van der Maas, H., Kan, K.-J., Marsman, M., Stevenson, C. E., 2017. Network models for cognitive development and intelligence. Preprints.

van der Maas, H. L., Dolan, C. V., Grasman, R. P., Wicherts, J. M., Huizenga, H. M., Raijmakers, M. E., 2006. A dynamical model of general intelligence: the positive manifold of intelligence by mutualism. Psychological review $113(4), 842-861$.

van der Maas, H. L., Kan, K.-J., Borsboom, D., 2014a. Intelligence is what the intelligence test measures. seriously. Journal of Intelligence 2 (1), 12-15.

van der Maas, H. L. J., Kan, K. J., Hofman, A. D., Raijmakers, M. E. J., 2014b. Dynamics of development: a complex systems approach. In: Molenaar, P. C. M., Lerner, R. M., Newell, K. M. (Eds.), Handbook of developmental systems theory and methodology. Guilford Press, New York, pp. 270-286.

van der Ven, S., Kroesbergen, E., Boom, J., Leseman, P., 2012. The development of executive functions and early mathematics: A dynamic relationship. British Journal of Educational Psychology 82 (1), 100-119.

van der Ven, S. H., Straatemeier, M., Jansen, B. R., Klinkenberg, S., Van der Maas, H. L., 2015. Learning multiplication: An integrated analysis of the multiplication ability of primary school children and the difficulty of single digit and multidigit multiplication problems. Learning and Individual Differences 43, $48-62$.

Verachtert, P., De Fraine, B., Onghena, P., Ghesquière, P., 2010. Season of birth and school success in the early years of primary education. Oxford Review of Education 36 (3), 285-306.

Wagenmakers, E.-J., Farrell, S., 2004. Aic model selection using akaike weights. Psychonomic Bulletin \& Review 11 (1), 192-196.

URL http://dx.doi.org/10.3758/BF03206482

Wohlwill, J. F., 1973. The study of behavioral development. Academic Press. 


\section{A Description of Math Garden}

Data was collected with an adaptive learning program for mathematics called Math Garden (www.mathsgarden.com; Klinkenberg et al., 2011; Straatemeier, 2014). After logging in, children arrive at a page showing a garden, where each plant represents a game that covers a particular math domain. A game starts after selecting the corresponding plant. Children are given 15 items to solve. They respond by clicking on a response option (counting and addition) or by using the virtual numeric keypad (multiplication and division). Each item is presented with a time limit of 20 seconds. The time is visualized by disappearing coins (one is lost each second that a response is not provided). If a correct response is given the coins are added to a money bag, but they are subtracted if the response is incorrect. The scoring rule rewards fast correct responses, but also punishes fast incorrect responses. The score $(S)$ that follows from this model is defined as follows:

$$
S=\left(2 X_{p i}-1\right)\left(d-T_{p i}\right),
$$

with the following expected scores, based on the current $\theta$ en $\beta$ estimates:

$$
E(S \mid \theta, \beta)=d \frac{\exp (2 d(\theta-\beta))+1}{\exp (2 d(\theta-\beta))-1}-\frac{1}{\theta-\beta}
$$

This explicit scoring-rule - called the High Speed High Stakes (HSHS) scoring rule (Klinkenberg et al., 2011; Maris and Van der Maas, 2012) - informs players on how to weigh speed and accuracy. This ensures that children perceive some time-pressure and are motivated to provide fast responses. But, they are also discouraged from guessing due to the penalty on a fast incorrect response. When a child does not know the answer (s)he can best wait the full 20 seconds. To prevent such waiting times the child can also use the question-mark button, in which case (s)he does not win or lose any coins.

With the HSHS scoring rule in the Math Garden the estimates of both the user ability and the item difficulty can be updated after each response using an Elo updating scheme (Elo, 1978):

$$
\begin{aligned}
\theta_{p} & =\theta_{p}+K_{p}\left(S_{p i}-E(S)_{p i}\right) \\
\beta_{i} & =\beta_{i}-K_{i}\left(S_{p i}-E(S)_{p i}\right) .
\end{aligned}
$$

This can be seen as a dynamic system where both $\theta$ and $\beta$ change can change over time. $K$ is a smoothing parameter that determines the variance in the ratings in the system. See Klinkenberg et al. (2011), Pelánek (2016) and Brinkhuis and Maris (2009) for more details about Elo in the context of adaptive testing.

Based on these estimates, relevant items are selected for each player at each time-point, such that children were expected to provide 60,75 or $90 \%$ correct responses when playing at the hard, medium or easy difficulty level (for more details see Jansen et al., 2016). For the current study we only selected children who played at the medium and hard difficulty levels and for which the average proportion of correct responses were around the aimed proportions, respectively .60 and .75. This ensured that the analyzed ability estimates were reliable indicators of the true skills at different periods. 


\section{B Simulation Study}

We performed different simulation studies to investigate two issues. First, we tested the recovery of the estimated coupling $(\gamma)$ and self-feedback $(\beta)$ parameters and of the correlational structure on the latent change factors $\left(\Delta X_{t}, \Delta X_{t+1}, \Delta Y_{t}, \Delta Y_{t+1}\right)$ under different scenario's. Second, we investigated the model comparison of the bidirectional coupling model (the full model), the no-coupling model and the $g$-factor model with varying strengths of the coupling parameters.

\section{B.1 Parameter recovery}
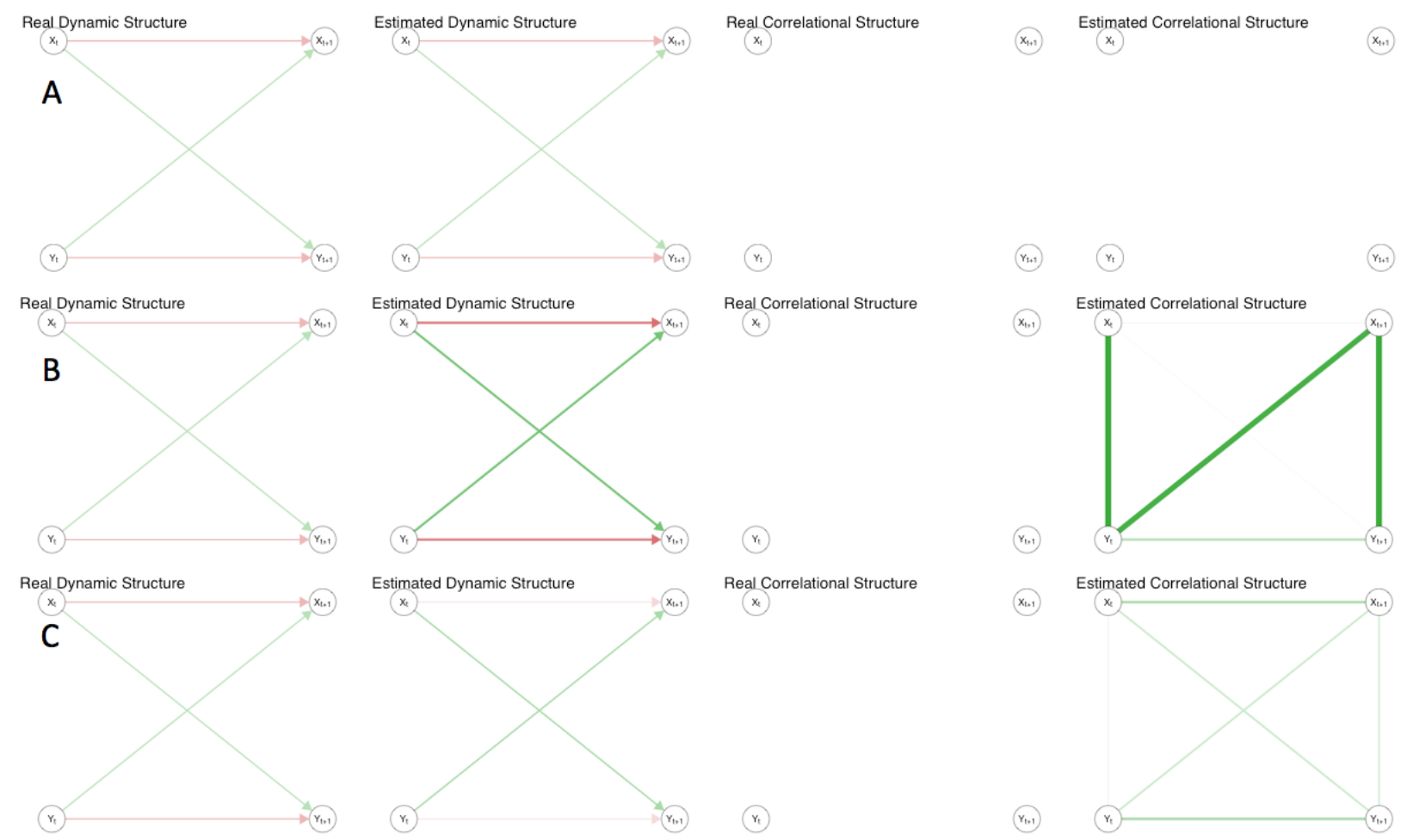

Figure 6: The results of the simulation study under three different scenario's: $\mathrm{A}=$ true model; $\mathrm{B}=$ selection of time-points; $\mathrm{C}=$ selection of variables. Here you see a simplified depiction, where only the relevant part of the full bivariate LCSM is shown. The circles represent change scores of variables $\mathrm{X}$ and $\mathrm{Y}$ at times $\mathrm{T}$ and $\mathrm{T}+1$. The first column shows the coupling (green; indicating a positive effect) and self-feedback (red; indicating a negative effect) effects of the true model and the second column shows the average estimated effects over the 500 runs. The third and fourth columns show the true correlational structure (zero in all true models) and estimated correlation structure respectively between the change factors. The plot was made with the qgraph package (Epskamp et al. 2012) in R. We set the maximum value of the edges to .7 to clearly depict the difference between the estimated model parameters.

We tested the parameter recovery of the correlations between the change scores in the bivariate latent change score model under three different scenarios: (1) parameter recovering in the true model, (2) selection of time-points, and (3) subset of variables. 500 data sets were simulated for each scenario with a bivariate dual latent change score model of three time-points and 5,000 participants. These settings were chosen to resemble the configurations of the data sets and the estimated model parameters of the current study. In the dynamic structure, the coupling parameters $(\gamma)$ and the feedback parameters $(\beta)$ were respectively set to .2 
and -.2 , the average of change score factors $(\Delta)$ to .5 with a variance of .3 . To test the effects of the different scenarios on the correlational structure we set the correlations between the change factors (correlational structure) to zero in the true model. The variance of the scores at t 1 were .5 and the error variances of all time-points were .1. The model specifications were equal to the models presented in section 2.1 , but to simplify the model we omitted the in this case unnecessary covariates (age and counts) at T1 because.

Scenario A: parameter recovery in the true model First, we investigated the recovery of the parameters under the true model. The results showed that under the specifications both the parameters of the dynamic and correlational structure we used were perfectly recovered, see upper-panel of Figure 6 .

Scenario B: selection of time-points Second, we investigated the effect of collecting a subset of the time-points on which the real developmental process unfold. To this end we generated data sets with seven time-points while only three time-points were observed and analyzed (the first, fourth and seventh time-point). Hence, in the true model multiple iterations were present between the dynamical structure of both coupled variables between the observed time-points. A comparison of the real and the estimated parameters in the dynamical structure (see middle-panel of Figure 6) shows that, as expected, these parameters were overestimated (on average the estimated parameters are around twice as large as the true parameters). More importantly, also the correlational structure is largely overestimated (with an average correlation of .32; varying between .03 and .5). This indicated that this correlational structure can merely result from a data selection problem, and an inflation of these correlations are expected if only a subset of the time-points of the true dynamical system are analyzed.

Scenario C: subset of variables Third, we investigated the effect of collecting just a subset of all variables that thrive the full dynamical system. Therefore, we again simulated 500 data sets based on a LSCM with four variables with the same configurations as discussed before. As expected, in the dynamical system the self-feedback parameters were underestimated (-.1 instead of -.2) and the coupling parameters were overestimated (.25 instead of .2). This can be explained by the positive relation with other variables that were not selected, although these variables did drive the changes scores upwards in the data simulation. Also, the correlational structure between the change scores was overestimated (correlations ranging from .06 to .24 with an average of .16). This simulation indicated that a significant correlational structure between the change scores in the LSCM can be caused by the analyses of just a small subset of the possibly larger set of relevant variables that thrive the changes in the dynamical system.

To conclude, the model shows very good parameter recovery under the true model, but both a selection of time-points and including only a subset of all variables of the real developmental system will result in an inflated correlational structure on the change scores variables. These results should be taken into account in the interpretation of such a correlational structure in a real data example. Crucially, the results of Scenario $\mathrm{A}$ and $\mathrm{B}$ show that under a true model with (positive) mutualistic effects and no correlations between change scores, a selection of time-points or analyses of a subset of all variables results in an overestimation of these correlations. This shows that in addition to a $g$-factor explanation of the correlational structure, these correlation can also be caused by a mutualistic account on development.

\section{B.2 Model comparison}

In a second simulation study we investigated the model comparison between the bidirectional coupling model (the full model), the no-coupling model and the $g$-factor model with varying strengths of the coupling parameters. We used the same set-up as in the previous simulation study, but with varying coupling parameters. These were sequentially set to $0, .05, .1, .15, .2, .25$ and .4 , ranging from absent to moderately high. This resulted in seven sets of 500 data sets. For each set we calculated the differences between AIC and BIC values for the comparison of the full model with coupling parameters, constrained model without coupling parameters and the $g$-factor model. The results are presented in Figure7, The left-panel plot shows that, as expected, the differences between the BIC values were higher for higher coupling parameters 

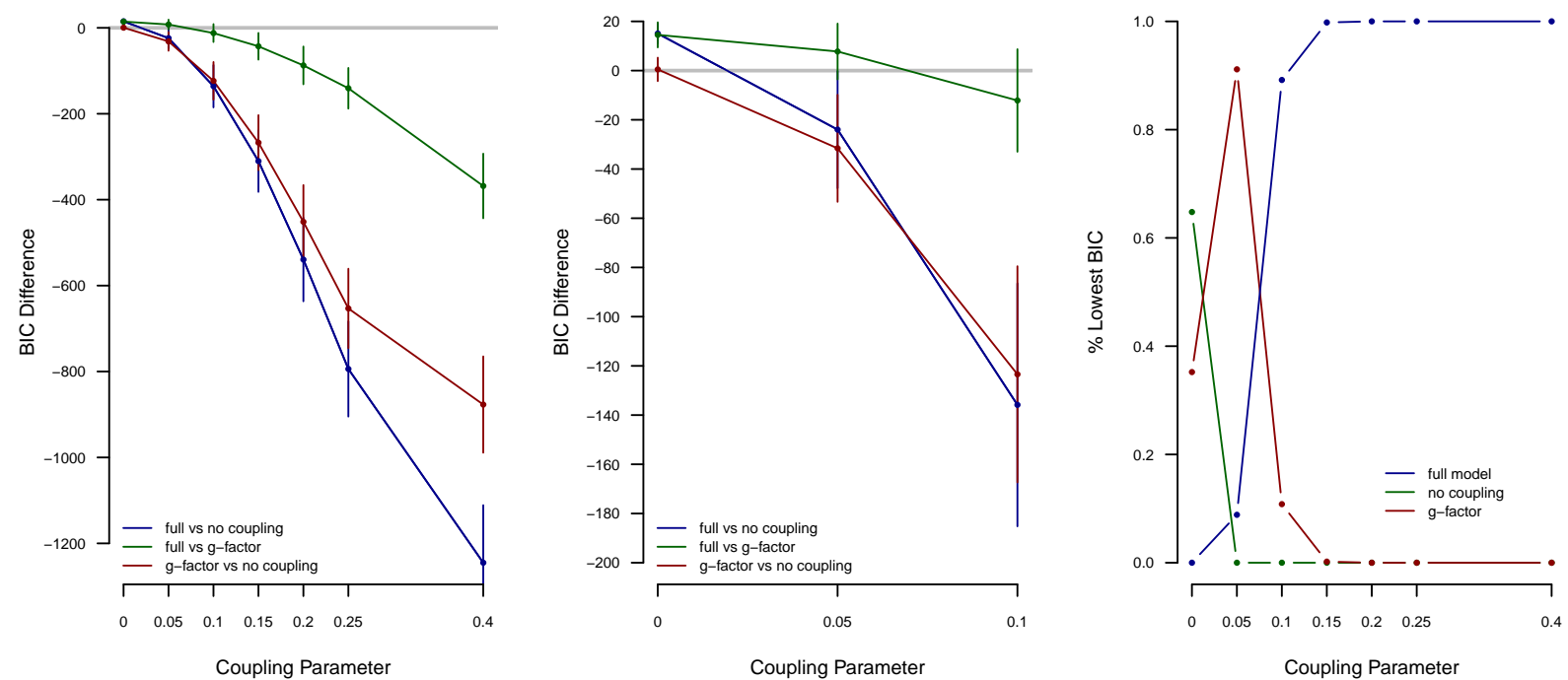

Figure 7: Model comparison using BIC differences based on simulated data sets of the bidirectional coupling model with varying coupling parameters.

(negative differences indicates that the first model has a lower BIC values than the second model and thus results in a better fit). The vertical lines indicate the standard deviations $(2 * \mathrm{SD})$ of the differences. Both the full and the $g$-factor model clearly outperform the no coupling model when the coupling parameter is higher than or equal to .1. This indicated that under the utilized data generation we have enough power to reliably classify coupling parameters of .1 as significantly different from zero. Also, as expected, the full model outperforms the $g$-factor model for high values of the coupling parameters. A close look at the results of the smaller coupling parameters (middle-panel) indicates that if the true coupling parameters are zero the BIC differences indicate that the full model is, as expected, outperformed by both the no-coupling and the $g$-factor model. Additionally, the BIC differences between the no-coupling model and the $g$-factor model are distributed around zero, which shows that in this case both models result in a comparable fit. This is also shown in the right-panel plot of Figure7 if the coupling parameters were set to zero, in about $60 \%$ of the runs the no-coupling model outperforms the other models, whereas the $g$-factor model is the best model in the other $40 \%$ of the runs. An unexpected result was the high performance of the $g$-factor model, outperforming the other models in $90 \%$ of the runs, when the coupling parameters were set to 05 . Thus under these settings the $g$-factor model outperforms both the no-coupling model and the full model. To conclude, both the no-coupling model and the $g$-factor model provide a good baseline model to test the presence of mutualistic coupling parameters and that the full model outperforms the other models under the current settings of the data simulation if such coupling is introduced (coupling values above .1). 


\section{Model parameters multiplication and division}

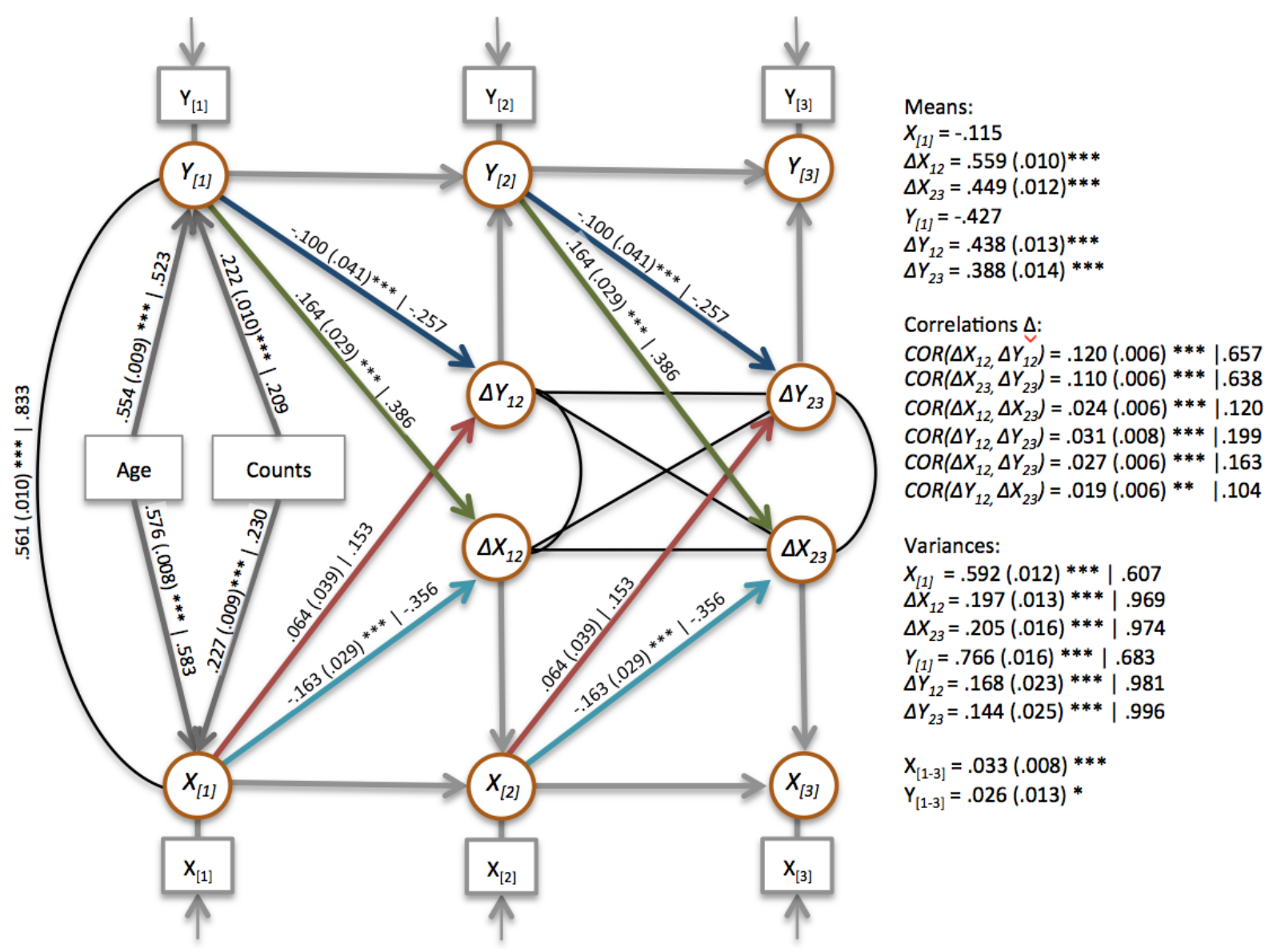

Figure 8: The estimated model parameters for the best fitting mutualism model in the multiplication and division data set. The first value denotes the unstandardized coefficient, with the standard error between brackets, and the value after the vertical bar denotes the standardized coefficient. The stars indicate the significant levels $\left(*=\mathrm{p}<.05,{ }^{* *}=\mathrm{p}<0.01\right.$ and $\left.{ }^{* * *}=\mathrm{p}<.001\right)$. The observed scores (X and $\left.\mathrm{Y}\right)$ are the latent trait estimates provided by Math Garden. 